\title{
Latest results from the NEMO-3 experiment and status of SuperNEMO
}

\author{
Karol Lang ${ }^{* \dagger}$ \\ The University of Texas at Austin \\ E-mail: lang@physics.utexas.edu
}

The NEMO-3 experiment, designed to search for neutrinoless double beta decay, was carried out from 2003 to 2011 in the Modane Underground Laboratory in the Fréjus Tunnel under the French-Italian Alps. The detector employed thin isotopic foils surrounded by a drift chamber and scintillator blocks to reconstruct topology, energy, and timing features of nuclear decays. This multi-observable technique offers a powerful means not only to identify double beta decays but also to reject background events mostly due to natural radioactivity. NEMO-3 employed seven different isotopes to construct foils, with most notable mass of ${ }^{100} \mathrm{Mo}$ of $6.9 \mathrm{~kg}$ and ${ }^{82} \mathrm{Se}$ of $0.93 \mathrm{~kg}$. Data from the entire running period are currently being analyzed but NEMO-3 has already achieved the best-to-date results on half-lives of all seven isotopes and has reported lower limits on neutrinoless double beta half-lives which can be translated to the most stringent upper limit on the effective neutrino mass.

SuperNEMO, the next generation experiment, will employ the technique pioneered by NEMO-3 but will ultimately house about $100 \mathrm{~kg}$ of an isotopic source distributed in 20 detector modules. SuperNEMO's goal is to reach half-life sensitivity of about $10^{26}$ years and thus about $50 \mathrm{meV}$ for an upper limit for the effective neutrino mass. The collaboration has conducted an extensive $\mathrm{R} \& \mathrm{D}$ program to improve detector performance and lower backgrounds in the new detector and the construction of the first "demonstrator" module has commenced. The module will be commissioned in 2014 and remaining modules will be built later in the decade. The baseline choice for an isotope is ${ }^{82} \mathrm{Se}$ but this technique has flexibility to use any other source. The collaboration also considers ${ }^{48} \mathrm{Ca}$ and ${ }^{150} \mathrm{Nd}$ if sufficient amounts of these isotopes can be enriched, currently viewed as an extremely challenging task of its own.

36th International Conference on High Energy Physics

4-11 July 2012

Melbourne, Australia

\footnotetext{
*Speaker.

$\dagger$ On behalf of the NEMO Collaboration which includes researchers from LAL Orsay, IPHC-DRS Strasburg, LPC Caen, CENBG Bordeaux, ITEP Moscow, JINR Dubna, INR Kiyev, University College London, University of Manchester, USMBA Fes, University of Texas at Austin, Idaho National Laboratory, Mount Holyoke College, IEAP Prague, Charles University Prague, Comenius University Bratislava Universidad de Valencia, Saga University, Osaka University, and Jyväskylä University.
} 


\section{Introduction}

The discovery of neutrino oscillations not only demonstrates that neutrinos have mass but also implies the existence of new symmetries beyond the Standard Model. These remarkable observations, perhaps more strongly than ever, motivate further investigations of properties of neutrinos. Some natural conjectures (e.g., seesaw mechanism [1]) suggest that deeper understanding of neutrinos may provide a glimpse onto phenomena at a Plank-scale energy level, could broaden our knowledge of the fundamental interactions, may shed light onto baryo- and lepto-genesis, and can help to better understand the early evolution of the Universe [2].

Majorana neutrinos [3], for which a neutrino and an antineutrino are represented by the same fundamental field, lead to a second-order weak process [4] illustrated by a diagram in Fig. 1. The left diagram shows a standard double-beta decay $(2 v \beta \beta)$, while in the right diagram, possible only if neutrinos are Majorana-type, the transition changes the lepton number by two units, $\Delta L=2$, so the resulting two electrons in the final state have a total energy equal to the energy of nuclear transition between nuclei $(A, Z)$ and $(A, Z+2)$. This seemingly simple feature provides the main observable for detecting $0 v \beta \beta$, and poses experimental challenges in searches for such processes.
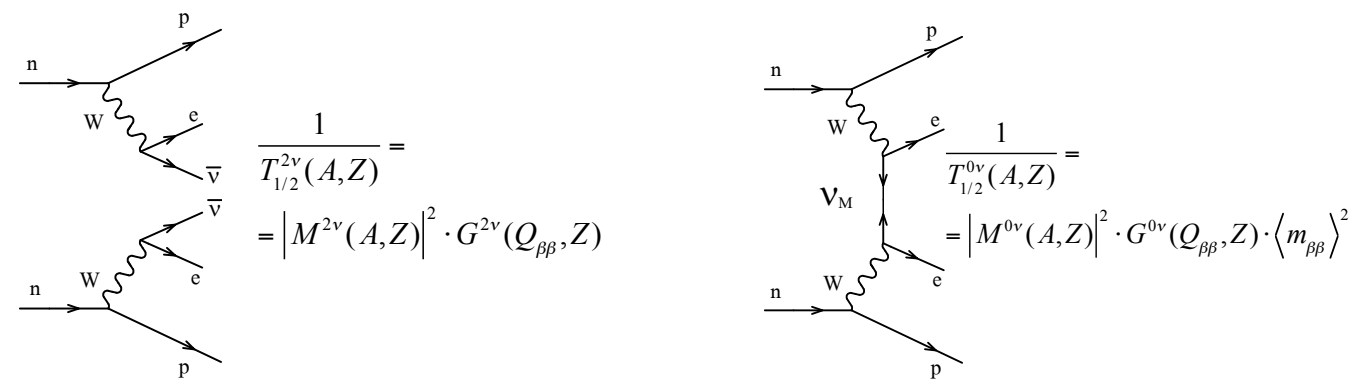

Figure 1: Feynman diagrams for two-neutrino (left) and neutrinoless double beta decay (right).

As depicted in Fig. 1, a half-life for $2 v \beta \beta$ and $0 v \beta \beta$ depends on the phase space factor, $G\left(Q_{\beta \beta}, Z\right)$, where $Q_{\beta \beta}$ is the $0 v \beta \beta$ transition energy, nuclear matrix element, $M(A, Z)$, and for $0 v \beta \beta$ also on the effective Majorana neutrino mass $\left\langle m_{\beta \beta}\right\rangle$, which is a sum of the mass eigenstates weighted by the squared elements of the PMNS neutrino mixing matrix [5], $\left\langle m_{\beta \beta}\right\rangle=\sum_{i=1,2,3} U_{e i}^{2} m_{i}$.

Presently, the best upper limit on $\left\langle m_{\beta \beta}\right\rangle$ comes from the EXO-200 [6] and KamlLAND-Zen [7] experiments with ${ }^{136} \mathrm{Xe}$ which recently reported $T_{1 / 2}^{0 v}>1.6 \times 10^{25} \mathrm{y}$ and $T_{1 / 2}^{0 v}>1.9 \times 10^{25} \mathrm{y}$, respectively. This gives a combined upper bound on $\left\langle m_{\beta \beta}\right\rangle<120-250 \mathrm{meV}$ [7]. The range of values for the $\left\langle m_{\beta \beta}\right\rangle$ upper limit reflects theoretical uncertainties in nuclear matrix elements [8]. This result effectively refutes the positive claim for ${ }^{76} \mathrm{Ge}[9]$.

Experiments face three practical challenges: suppression of backgrounds due to natural radioactivity, achieving detector performance (most importantly good energy resolution) to identify the final state transition of $0 v \beta \beta$, and attaining large exposure (mass $\times$ time), which usually requires isotopic enrichment. There is no obvious path how to accomplish high sensitivity to $0 v \beta \beta$. Experiments "bet" on different detector technologies. The NEMO-3 technique is quite unique: it employs a tracker and a calorimeter to obtain information on timing, topology, and kinematics of particles in the final state. This multi-observable approach not only allows to identify the signal but it is also capable of measuring most background processes. 


\section{NEMO-3 and SuperNEMO}

The NEMO-3 experiment [10] - a search for $0 v \beta \beta$ - was carried out from 2003 to 2011. The detector was located in the Modane Underground Laboratory under about $1,700 \mathrm{~m}$ of rock $(4,800 \mathrm{~m}$ water-equivalent) in the Fréjus Tunnel under the French-Italian Alps. The NEMO Collaboration also conducted an extensive $\mathrm{R} \& \mathrm{D}$ program to design the next phase of the experiment, SuperNEMO, with the sensitivity to Majorana neutrino mass in the $50 \mathrm{meV}$ range.

The NEMO-3 detector had a cylindrical geometry with a $6 \mathrm{~m}$ diameter and $3 \mathrm{~m}$ height, as shown in Fig. 2. It was divided into 20 azimuthal sectors, each housing a foil with one or more of seven enriched isotopes. The main isotopes featured in the experiment were ${ }^{100} \mathrm{Mo}$ and ${ }^{82} \mathrm{Se}$ but, in addition, five other isotopes $\left({ }^{130} \mathrm{Te},{ }^{96} \mathrm{Zr},{ }^{150} \mathrm{Nd},{ }^{116} \mathrm{Cd},{ }^{48} \mathrm{Ca}\right)$ were employed in smaller quantities. The experiment used isotopic foils surrounded by a wire drift chamber (operated in a Geiger mode) for 3D tracking in a $25 \mathrm{G}$ magnetic field, and large scintillator blocks with photomultipliers for calorimetric measurement of electron energies. The detector made it possible to reconstruct the topology, energy, and timing features of nuclear decays. This technique delivers several observables for each event, providing a powerful means not only to identify double beta decays but also to measure and reject background events $[10,11]$.

The NEMO-3 apparatus was turned off on January 11, 2011, after collecting data for almost eight years. The experiment continued operating well until the end but reached a point of diminishing returns. The decision to turned It off was based on operating costs, and the need to prepare the cavern for the SuperNEMO Demonstrator module. The dismantling of the NEMO-3 detector is now completed and the cavern is being outfitted for SuperNEMO.
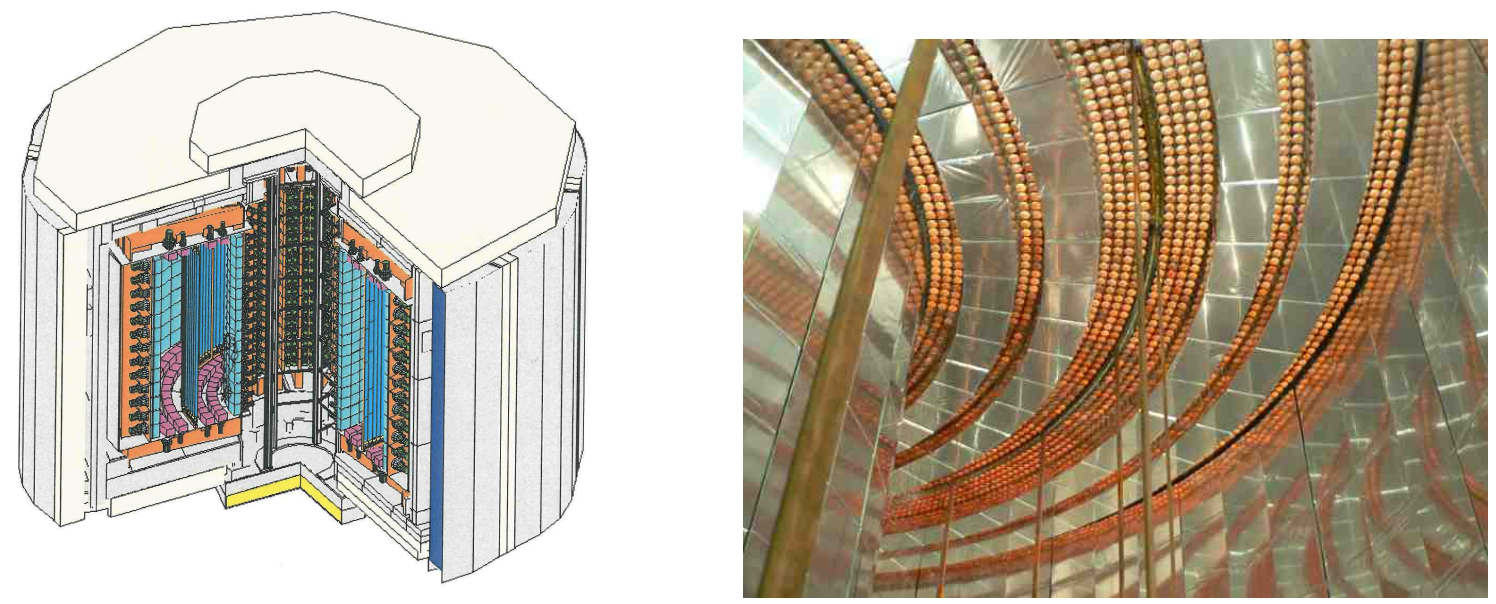

Figure 2: Left: A schematic view of the NEMO-3 detector. Right: A pictures of an interior of the NEMO-3 detector (taken during the decommissioning in May 2011).

SuperNEMO will further exploit the NEMO-3 technique. The new detector will be planar and its most essential features will be substantially improved, as shown in Fig. 3. A full detector will have 20 such modules, each with a fiducial area of $5 \times 3 \mathrm{~m}^{2}$ and each housing $5 \mathrm{~kg}$ of ${ }^{82} \mathrm{Se}$. If larger quantities of ${ }^{48} \mathrm{Ca}$ or ${ }^{150} \mathrm{Nd}$ become available, SuperNEMO would deploy these isotopes in new modules. (Isotopes of ${ }^{48} \mathrm{Ca}$ and ${ }^{150} \mathrm{Nd}$ are desirable due to their high $Q_{\beta \beta}$ value thus easier background suppression. However, the enrichment of both is extremely difficult, although new 

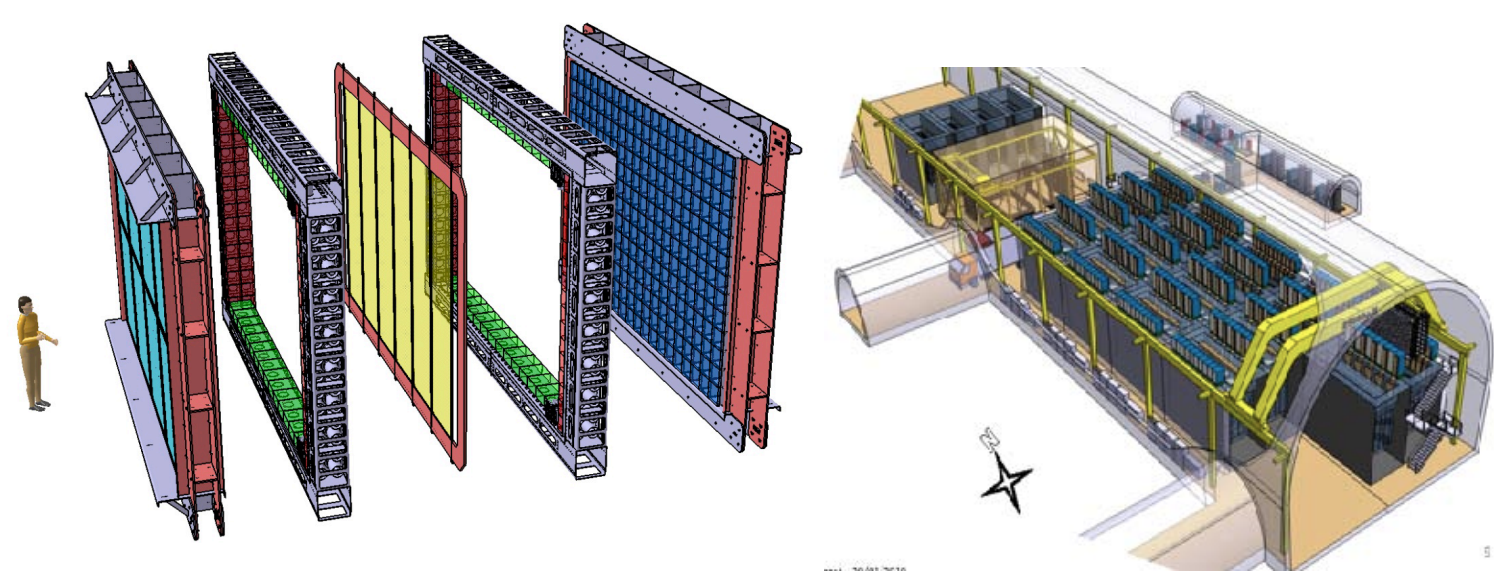

Figure 3: Left: A schematic view of the main inner parts of the SuperNEMO Demonstrator module. The two scintillator calorimeter walls surround the tracking chambers. The central frame, holding the isotopic source foil, is installed between the two tracker halves. This core part will be surrounded by scintillators on top and bottom, and sides for hermetic gamma coverage, and by passive shielding of iron, water, and radon-filtered air. Right: SuperNEMO's cavern (housing 20 modules).
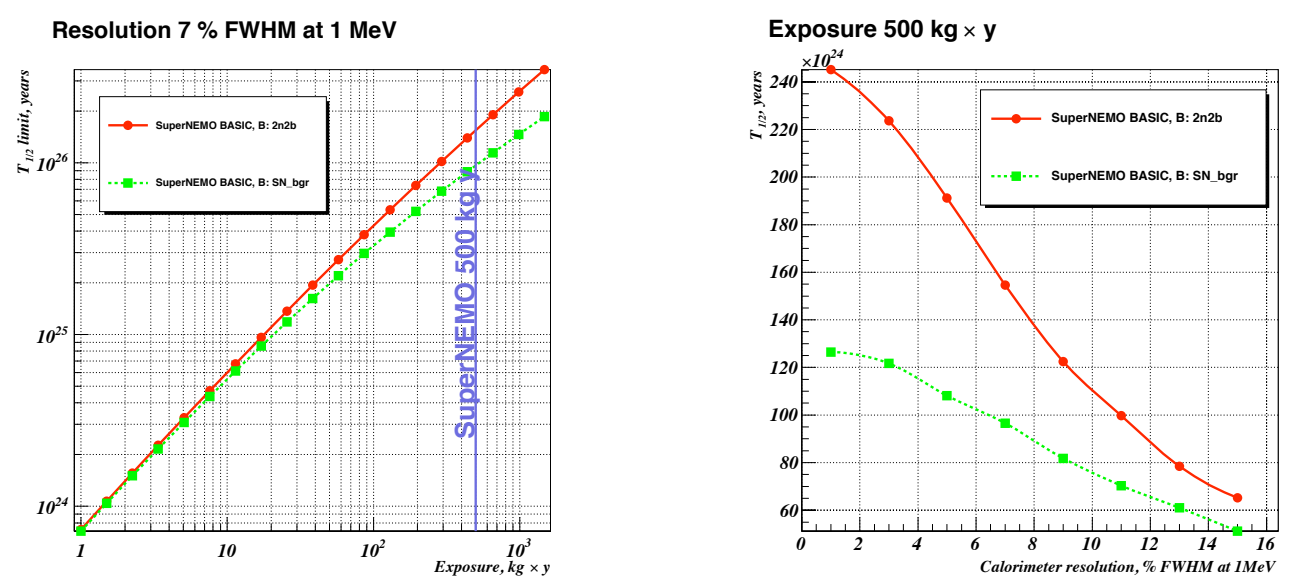

Figure 4: The SuperNEMO exposure is planned for $500 \mathrm{~kg}$-years which should reach sensitivity of $1 \times 10^{26}$ (left plot). It will somewhat depend on the effective energy resolution of the calorimeter (right plot).

technologies may be emerging.) The goal of SuperNEMO is to probe the ${ }^{82} \mathrm{Se}$ half-life for $0 v \beta \beta$ at the level of $1 \times 10^{26}$ in a $500 \mathrm{~kg}$-year exposure, as illustrated in Fig. 4, corresponding to the $\left\langle m_{\beta \beta}\right\rangle$ sensitivity in the $50 \mathrm{meV}$ range.

The first SuperNEMO module, Demonstrator, will be slightly different than modules foreseen for the future. It will house about $7 \mathrm{~kg}$ of ${ }^{82} \mathrm{Se}$ and will be constructed in the location of the NEMO-3 apparatus. Future modules will be located in the recently approved extension of the Modane laboratory. The construction of the Demonstrator has started and the beginning of its operations is projected for 2014. With a two-year exposure, the Demonstrator should reach the half-life sensitivity of $2 \times 10^{25}$. 


\section{Highlights of NEMO-3 results}

The NEMO-3 experiment took data almost continuously for about eight years between 2003 and 2011. The ever increasing data set has been analyzed during the data-taking period but no results have been yet obtained with a full exposure. NEMO-3 has published preliminary results on life-times of all isotopes in its use. For ${ }^{100} \mathrm{Mo}$ (shown in Fig. 5) and ${ }^{82} \mathrm{Se}$, the "flagship" isotopes, because of their total mass in the detector of nearly $7 \mathrm{~kg}$ and $1 \mathrm{~kg}$, respectively, the experiment reported [12]: $T_{1 / 2}^{2 v}=[7.16 \pm 0.01$ (stat) $\pm 0.54($ syst $)] \times 10^{18}$ y for ${ }^{100} \mathrm{Mo}$, and $T_{1 / 2}^{2 v}=$ $[9.6 \pm 0.1$ (stat) \pm 1.0 (syst) $] \times 10^{19}$ y for ${ }^{92} \mathrm{Se}$. NEMO-3 has also reported [12] preliminary lower limits on $0 v \beta \beta$ half-lives: $T_{1 / 2}^{0 v}>1.0 \times 10^{24} \mathrm{y}$ for ${ }^{100} \mathrm{Mo}$ and $T_{1 / 2}^{0 v}>3.2 \times 10^{23} \mathrm{y}$ for ${ }^{82} \mathrm{Se}$. The lower limit from ${ }^{100}$ Mo translates to $\left\langle m_{\beta \beta}\right\rangle<310-960 \mathrm{meV}$, where the spread reflects uncertainties in the nuclear matrix element.
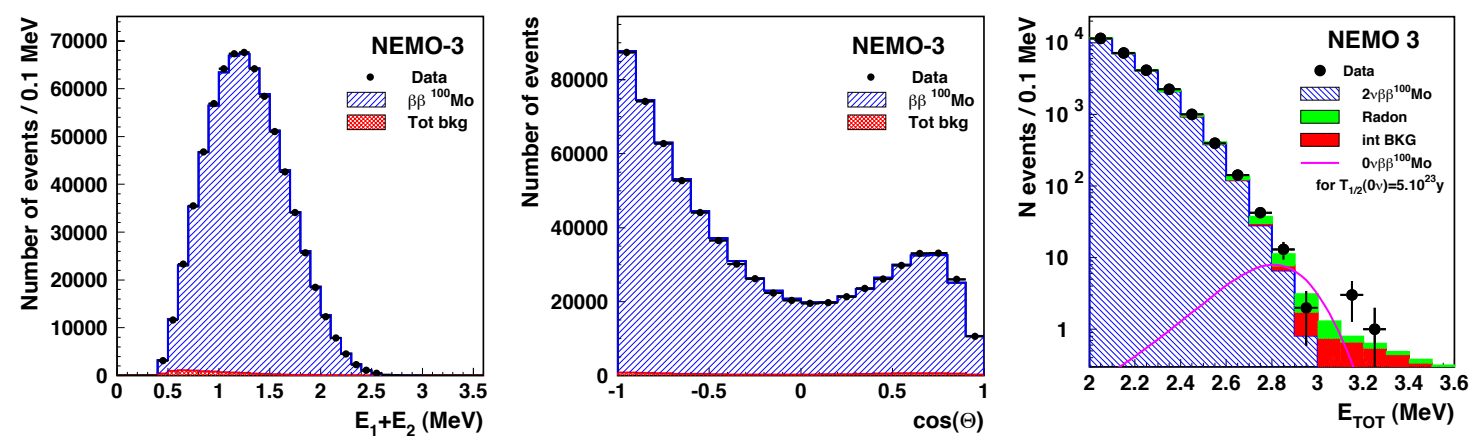

Figure 5: NEMO-3 "flagship" measurement of $0 v \beta \beta$ and $2 v \beta \beta$ for ${ }^{100}$ Mo. The left plot shows the energy distribution of two electrons in more than 700,000 candidate $2 v \beta \beta$ events, the middle plot shows the angular distribution between the two electrons, and the right plot is a zoomed-in plot on the left near the end-point or transition energy of 3.034 MeV. For ${ }^{100} \mathrm{Mo}$ foil the $2 v \beta \beta$ background is almost negligible. Events above $3 \mathrm{MeV}$ are background events largely due temporal PMT instabilities. A Monte Carlo simulation of $0 v \beta \beta$ shown as a pink line is for a hypothetical $T_{1 / 2}^{0 v}=5 \times 10^{23} \mathrm{y}$.

\begin{tabular}{|l|r|c|c|c|c|c|}
\hline Isotope & $\begin{array}{r}\text { Mass } \\
(\mathrm{g})\end{array}$ & $\begin{array}{c}Q_{\beta \beta} \\
(\mathrm{keV})\end{array}$ & $\begin{array}{c}T_{1 / 2}(2 v \beta \beta) \\
\left(\times 10^{19} \mathrm{y}\right) \\
( \pm \text { stat. } \pm \text { syst. })\end{array}$ & $\begin{array}{c}T_{1 / 2}(0 v \beta \beta) \\
90 \% \text { C.L. }(\mathrm{y})\end{array}$ & $\begin{array}{c}\left\langle m_{\beta \beta}\right\rangle \\
90 \% \mathrm{C} . \mathrm{L} . \\
(\mathrm{eV})\end{array}$ & Ref. \\
\hline${ }^{100} \mathrm{Mo}$ & 6,914 & 3,035 & $0.716 \pm 0.001 \pm 0.054$ & $>1.0 \times 10^{24}(*)$ & $<0.31-0.96$ & {$[12,13]$} \\
${ }^{82} \mathrm{Se}$ & 932 & 2,995 & $9.6 \pm 0.1 \pm 1.0$ & $>3.2 \times 10^{23}(*)$ & $<0.94-2.6$ & {$[12,13]$} \\
${ }^{130} \mathrm{Te}$ & 454 & 2,529 & $70 \pm 9 \pm 11$ & $>1.3 \times 10^{23}$ & $<1.3-3.6$ & {$[14]$} \\
${ }^{116} \mathrm{Cd}$ & 405 & 2,805 & $2.88 \pm 0.04 \pm 0.16$ & $>1.3 \times 10^{23}$ & $<1.3-3.2$ & {$[12,15]$} \\
${ }^{150} \mathrm{Nd}$ & 37.0 & 3,367 & $0.911_{-0.022}^{+0.025} \pm 0.063$ & $>1.8 \times 10^{22}(*)$ & $<1.5-6.8$ & {$[16]$} \\
${ }^{96} \mathrm{Zr}$ & 9.4 & 3,350 & $2.35 \pm 0.14 \pm 0.16$ & $>9.2 \times 10^{21}(*)$ & $<7.2-19.5$ & {$[13]$} \\
${ }^{48} \mathrm{Ca}$ & 7.0 & 4,272 & $4.4_{-0.4}^{+0.5} \pm 0.4$ & $>1.3 \times 10^{22}$ & $<29.6$ & {$[12]$} \\
\hline
\end{tabular}

Table 1: Summary of current NEMO-3 results for $T_{1 / 2}(2 v \beta \beta)$ and $T_{1 / 2}(0 v \beta \beta)$. Shown is also the used mass and the value of transition energy $Q_{\beta \beta}$ for each isotope. The uncertainty in the matrix element is reflected in the range of upper limits for the effective Majorana neutrino mass $\left\langle m_{\beta \beta}\right\rangle$. The symbol (*) indicates the current best upper limit for this process.

Table 1 summarizes the main features of isotopes used by NEMO-3 and the current results on $2 v \beta \beta$ half-lives and lower limits on $0 v \beta \beta$ transitions for these isotopes. It is worth to emphasize 
that, although these results are not yet based on the full exposure, four isotopes give the most stringent results ever reported. The most recent publication by NEMO-3 [14] presents a first real time measurement of the $2 v \beta \beta$ decay of ${ }^{130} \mathrm{Te} \rightarrow{ }^{130} \mathrm{Xe}$ with the half-life $T_{1 / 2}^{2 v}=[7.0 \pm 0.9$ (stat) \pm 1.1 (syst) $] \times 10^{20} \mathrm{y}$. This result firmly establishes this transition which was earlier inferred only through geochemical analysis.

\section{Outlook}

The first SuperNEMO Demonstrator module is currently under construction and will be commissioned in 2014. Concurrently, the full NEMO-3 data set is being analyzed and updated results on all seven isotopes will be published over the next two years. They will include searches for transitions to excited states and for Majoron transitions.

Acknowledgements: This work was supported by the US NSF and DOE, the French IN2P3, the UK STFC, and grants in Russia, Japan, the Czech Republic, and Slovakia. We are grateful to the crew of the Modane Underground Laboratory for their contribution to this effort.

\section{References}

[1] M. Gell-Mann, P. Ramond, R. Slansky, Conf. Proc. C790927, 315-321 (1979); T. Yanagida, Prog. Theor. Phys. 64, 1103 (1980); R. N. Mohapatra, G. Senjanovic, Phys. Rev. Lett. 44, 912 (1980).

[2] M. Fukugita and T. Yanagida, Phys. Lett. B 174, 45 (1986); R. N. Mohapatra and A. Y. Smirnov, Ann. Rev. Nucl. Part. Sci. 56, 569 (2006).

[3] E. Majorana, Nuovo Cim. 14, 171 (1937).

[4] J. Schechter, J. W. F. Valle, Phys. Rev. D25, 2951 (1982).

[5] B. Pontecorvo, Sov. Phys. JETP 34, 247 (1958); Z. Maki, M. Nakagawa, and S. Sakata, Prog. Theor. Phys. 28, 870 (1962).

[6] M. Auger et al. [EXO Collaboration], Phys. Rev. Lett. 109, 032505 (2012).

[7] A. Gando et al. [KamLAND-Zen Collaboration], arXiv:1211.3863 [hep-ex].

[8] A. Faessler et al., Phys. Rev. D 79, 053001 (2009).

[9] H. V. Klapdor-Kleingrothaus and I. V. Krivosheina, Mod. Phys. Lett. A 21, 1547 (2006).

[10] R. Arnold et al., [NEMO-3 Collaboration], Nucl. Instrum. Meth. A536, 79-122 (2005).

[11] J. Argyriades et al., [NEMO-3 Collaboration], Nucl. Instrum. Meth. A606, 449-465 (2009).

[12] L. Simard [for the NEMO Collaboration], TAUP 2011, 5-9 September 2011, Munich, Germany.

[13] R. Arnold et al., [NEMO-3 Collaboration], Phys. Rev. Lett. 95, 182302 (2005).

[14] R. Arnold et al., [NEMO-3 Collaboration], Phys. Rev. Lett. 107, 062504 (2011).

[15] R. Benton Pahlka, Ph.D. Thesis, University of Texas, August 2010.

[16] J. Argyriades et al. [NEMO-3 Collaboration], Phys. Rev. C80, 032501 (2009). 